pp. A Coruña.

HERRERO CEMBRANOS , L., M. E. GARCÍA GONZÁLEZ y A. PENAS MERINO -1988- De Plantis legionensibus. Notula XI. Acta Bot. Malacitana 13: 319-323.

LAÍNZ, M. -1976- Aportaciones al conocimiento de la flora cántabro-astur, XI. Bol. Inst. Est. Ast., ser. C, 22: 3-44.

MORALES VALVERDE, R. - 1986- Taxonomía de los géneros Thymus (excluida la Sección Serpyllum) y Thymbra en la Península Ibérica. Ruizia 3: 5-324.

PENAS MERINO, A. -1984- Nuevos taxones para la flora leonesa. Lagascalia 13(1): 3-16.

ROMERO RODRÍGUEZ, C. M. -1983- Flora y vegetación de la cuenca alta del río Luna (León). ICONA Monografías 29: 5-273.

TALAVERA, S. y B. VALDÉS -1976- Revisión del género Cirsium (Compositae) en la Península Ibérica. Lagascalia 5(2): 127-223.
TUTIN, T. G., V. H. HEYWOOD, N. A. BURGES, D. M. MOORE, D. H. VALENTINE, S. M. WALTERS \& D. A. WEBB (eds.). -1968-76Flora Europaea. Vol. II, III, IV. Cambrigde University Press.

Aceptado para su publicación en Abril de 1999

Dirección de los autores. Departamento de Biología Vegetal. Universidad de León. Campus de Vegazana s/n. E-24071. León. España.

\title{
79. NUEVAS LOCALIDADES ALICANTINAS DE CARDUNCELLUS DIANIUS WEBB
}

\author{
María Rosa SOLER, José Carlos CRISTÓBAL y Manuel B. CRESPO
}

New alicantine localities of Carduncellus dianius Webb

Palabras clave: Corología, flora, Alicante, España.

Key words: Chorology, flora, Alicante, Spain.

Durante la realización de diversos estudios sobre la flora y la vegetación de las sierras del norte de la provincia de Alicante, se ha constatado la existencia en la Serra de Bèrnia de dos nuevas localidades del interesante endemismo diánico-pitiúsico Carduncellus 




Figura 1. Distribución de Carduncellus dianius en el noroeste de Alicante ( localidades conocidas; $\star$ : nuevas localidades)

dianius Webb [ $\equiv$ Lamottea diania (Webb) G. López], que hasta ahora sólo se conocía de contadas localidades de la sierra del Montgó (Denia-Jávea) y sus proximidades. Con ello se amplía considerablemente hacia el sur la distribución de esta notable planta.

El tratamiento taxonómico de las plantas citadas se ajusta al propuesto por Mateo \& Crespo (1998). Los pliegos testigo del material citado están depositados en el herbario $\mathrm{ABH}$ de la Universidad de Alicante.

Carduncellus dianius Webb [\$ Lamottea diania (Webb) G. López]

ALICANTE: Callosa d'En Sarrià, Serra de Bèrnia, Penyal de l'Ovenga, 30SYH5583, 550 m, 8-6-1997, M.R. Soler \& J.C. Cristóbal (ABH 42104). Callosa d'En Sarrià, Serra de Bèrnia, pr. Penya Severino, 30SYH5484, 400 m, 6-4-1999, M.R. Soler \& J.C. Cristóbal (ABH 42109). Ampliación de área.

Este interesante endemismo se conoce de unas pocas localidades del extremo noreste de la provincia de Alicante y noroeste de Ibiza (cf. Bolòs \& Vigo, 1996). Concretamente, en Alicante se conocen poblaciones importantes en el Parque Natural del Montgó (Denia-Jávea) -de donde fue descrito por Webb (1838)- y en su zona periférica meridional (cf. Rigual, 1972; Pérez Badia, 1997; Barber, 1999), donde suele instalarse en áreas umbrosas y húmedas de los cantiles o pie de cantiles calizos cretácicos. Por ello, las nuevas localidades (fig. 1) amplían en más de $20 \mathrm{~km}$ hacia el sur su distribución peninsular conocida (cf. Laguna et al., 1998).

En las nuevas localidades, $C$. dianius muestra un comportamiento ecológico similar al resto, aunque en ambas su óptimo se encuentra en escarpes de roquedos calizos con un microclima particularmente húmedo y cálido. Fitosociológicamente participa en la Hippocrepido valentinae-Scabiosetum saxatilis Rivas Goday ex O. Bolòs 1957 (Teucrion buxifolii Rivas Goday in Rivas Goday et al. 1955). Un inventario tomado en la primera de las localidades citadas $\left(150 \mathrm{~m}^{2}, 70 \%\right.$ cobertura, $\left.85^{\circ} \mathrm{W}\right)$ refleja la siguiente composición florística: Scabiosa saxatilis Cav. 3.3, Carduncellus dianius Webb 2.2, Teucrium buxifolium Schreb. subsp. hifacense (Pau) Fern Casas 2.2, Asperula paui Font Quer subsp. dianensis (Font Quer) Romo 1.2, Chiliadenus glutinosus (L.) Fourr. 1.2, Melica minuta L. 1.2, Elaeoselinum asclepium (L.) Bertol. 1.2, Piptatherum coerulescens (Desf.) P. Beauv 1.2, Reseda valentina (Pau) Pau ex Cámara + .2, Galium fruticescens Cav. +.2, Chamaerops humilis L. +.2 , Centaurea rouyi Coincy + Sanguisorba ancistroides (Desf.) A. Br. + Biscutella montana Cav. +, Asplenium petrarchae (Guérin) DC. +, Lapiedra martinezii Lag. +, Sedum sediforme (Jacq.) Pau var. dianium (O. Bolòs) O. Bolòs + , Dianthus broteri Boiss. \& Reut. subsp. valentinus (Willk.) Rivas Mart. et al. +, Phagnalon saxatile (L.) Cass. +, Ceterach officinarum Willd. +, Polygala rupestris Pourr. +, y Asplenium fontanum (L.) Bernh. +. Además, de manera ocasional, algunos ejemplares de 
Carduncellus dianius penetran en fragmentos de los matorrales subrupícolas de Teucrio glauci-Hippocrepidetum valentinae $\mathrm{O}$. Bolòs 1956 (Asparago albi-Rhamnion oleoidis Rivas Goday ex Rivas Mart. 1975), que se instalan en las repisas anchas de tales roquedos. Allí se acompaña, además, de Teucrium flavum L. subsp. glaucum (Jord. \& Fourr.) Ronninger, Pistacia terebinthus L., P. lentiscus L., Rhamnus alaternus L., Coronilla juncea L., Fraxinus ornus L., Ceratonia siliqua L., Asparagus acutifolius L., Arbutus unedo L., Lonicera implexa Ait., Smilax aspera L., Hedera helix L., Euphorbia squamigera Loisel., Bupleurum fruticescens L., Erica multiflora L., Ruta angustifolia Pers., Rosmarinus officinalis L., Rubus ulmifolius Schott, Osyris lanceolata Hochst. \& Steud., Clematis flammula L. y Phillyrea latifolia L., entre otras.

En total, se han contabilizado cerca de 500 ejemplares, que florecen y fructifican con normalidad. Morfológicamente no presentan diferencias notables respecto al resto de plantas alicantinas, pudiendo asimilarse sin dificultad al tipo de la especie.

Dado que esta especie cuenta con poblaciones importantes en Alicante e Ibiza, Laguna \& Crespo (1996) han propuesto considerarla "Planta Vulnerable" (categoría VU de la U.I.C.N., noviembre de 1994). Sin embargo, ya que se halla incluida en el Anexo II -Especies y subespecies de "interés especial"del "Catálogo Nacional de Especies Amenazadas" (B.O.E. n ${ }^{\circ} 82$, de 5 de mayo de 1990), deberían establecerse medidas urgentes para su conservación. El establecimiento de una microrreserva de flora (cf. Laguna, 1996) en el Penyal de l'Ovenga podría ser una solución adecuada. Mientras tanto, deberían buscarse nuevas poblaciones en las sierras situadas entre los extremos conocidos de su área alicantina.

\section{BIBLIOGRAFÍA}

BARBER, A. -1999- Contribució al coneixement florístic i fitogeogràfic del litoral de la comarca de la Marina Alta (País Valencià). Premis 25 d'abril de 1995. Benissa.

BOLÒS, O. y J. VIGO -1996- Flora dels Països Catalans 3. Barcino. Barcelona.

LAGUNA, E. -1996- Conservación in situ mediante microrreservas de flora en la Comunidad Valenciana. Bol. Real Soc. Esp. Hist. Nat. Tomo Extraord. 125 Aniversario: 379-381. Madrid.

LAGUNA, E. y M.B. CRESPO -1996- Asignación de las nuevas categorías UICN a la flora endémica de la Comunidad Valenciana. Bol. Real Soc. Esp. Hist. Nat. Tomo Extraord. 125 Aniversario: 385387. Madrid.

LAGUNA, E., M.B CRESPO, G. MATEO, S. LÓPEZ UDIAS, S. FABREGAT, L. SERRA, J.J. HERRERO-BORGOÑÓN, J.L. CARRETERO, A. AGUILELLA y R. FIGUEROLA -1998- Flora endémica, rara o amenazada de la Comunidad Valenciana. Colecc. Biodiversidad n ${ }^{\circ} 1$. Generalitat Valenciana.

MATEO, G. y M.B. CRESPO-1998-Manual para la determinación de la flora valenciana. Monogr. Fl. Montiber. 3. Valencia.

PÉREZ BADIA, R. -1997-Flora vascular y vegetación de la comarca de la Marina Alta. Publ. Inst. Cult. Gil-Albert. Alicante

RIGUAL, A. -1972-Flora y vegetación de la provincia de Alicante. Inst. Estud. Alicantinos. Alicante.

WEBB, P.B. -1838-Iter hispaniense. París-Londres.

Aceptado para su publicación en Septiembre de 1999

Dirección de los autores: Departamento de Ciencias Ambientales y Recursos Naturales (Botánica). Universidad de Alicante. Apdo. 99. E-03080 Alicante. 